\title{
A versatile memory-mapped I/O interface for microcomputers
}

\author{
NAOYUKI OSAKA \\ Otemon-Gakuin University, Ibaraki, Osaka 567, Japan
}

\begin{abstract}
An inexpensive and easy-to-build input/output interface capable of driving the electromechanical loads (relays, solenoids, dc motors, etc.) and incandescent lamps is described. The interface hardware is based on the Commodore VIC-20 microcomputer. The design is adaptable to most 8-bit microcomputers.
\end{abstract}

Recent developments in microcomputer interfacing technology make real-time communication and control of experiments incredibly inexpensive (Osaka, 1980, 1982; Parks, 1978; Sidowski, 1978). A one-chip programmable interface large-scale integrated circuits (LSI) makes design of the psychological instrumentation relatively simple and inexpensive. However, an additional high-current/high-voltage power integrated circuit (IC) is needed to displace discrete components in tough applications. An evolution of high-current/high-voltage interface ICs is underway to provide answers for lowspeed electromechanical loads. One can easily build an intelligent parallel communication interface with little electronics knowledge by using one-chip peripheral versatile interface adapter (VIA) coupled with onechip Darlington drivers.

Commodore's VIC-20 (called VIC-1001 in Japan) BASIC microcomputer (6502A CPU with $20 \mathrm{~K}$ ROM and 5K RAM) is an inexpensive (\$317) and portable (1.8-kg) BASIC system (Commodore Business Machines, Inc., 1981). Further, VIC has a software/hardware compatibility with the PET/CBM series microcomputers.

\section{INTERFACING HARDWARE}

The VIC-20 has 8-bit parallel programmable $\mathrm{I} / \mathrm{O}$ lines for laboratory interfacing in which TTL-compatible I/O lines (PB0 to PB7 and PA2 to PA5) of the MCS6522VIA remain free as the parallel "user port" (Osaka, 1979) and are directly accessible using POKEing and PEEKing at each $\mathrm{I} / \mathrm{O}$ register address within a VIA chip. These 8-bit programmable I/O lines are on the VIC's main logic board and are terminated at the board edge (CN1). Sixteen memory-mapped register addresses (from 37136 to 37151 ) are allocated for this built-in VIA chip. These $\mathrm{I} / \mathrm{O}$ lines make the interfaces for connecting switch inputs and outputs to power drivers (i.e., relays, lamps, etc.) simple and inexpensive. Figure 1 indicates

This work was supported in part by Grant 56510078 from the Japan Ministry of Education and Yoshida Memorial Foundation.

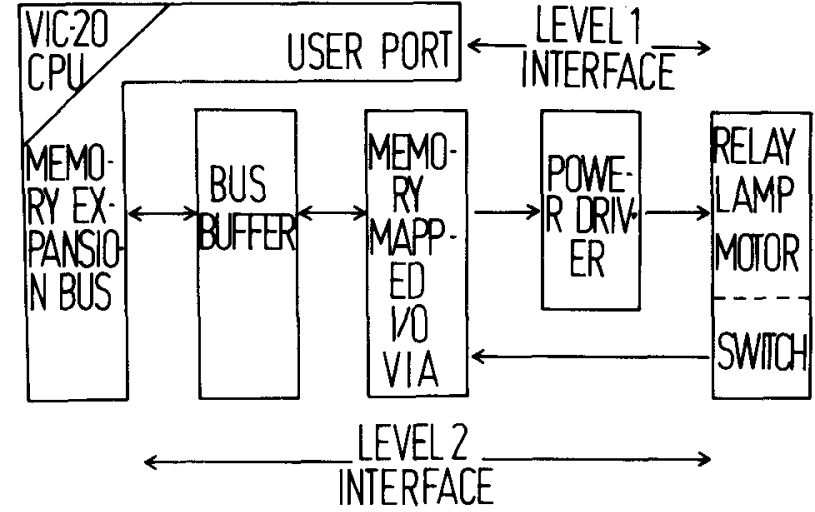

Figure 1. Level 1 and Level 2 peripheral $I / O$ interfaces: Level 1 interface requires only power driver and load; Level 2 requires additional bus buffers and external "memory-mapped" versatile interface adapter (VIA).

two ways to make general-purpose interfaces. The Level 1 and Level 2 interfaces use built-in parallel user port and memory expansion bus lines, respectively. The user port is buffered, but the memory expansion bus line is not.

\section{Level 1 Communication Interface}

The Level 1 interface is easy to make, but the microcomputer must have memory-mapped parallel $\mathrm{I} / 0$ ports (as the VIC does). Figure 2 provides an illustration of hardware connecting a user port to several loads. As an example, the PB0-PB5 ports and PB7 port are used for output and input, respectively. The logic buffer inverters (LS04 $\times 2$ ) drive both the light-emitting diodes (LEDs) and the Darlington transistors. Sprague's ULN$2003 \mathrm{~A}$ chip has high-current $\left[\mathrm{I}_{\mathrm{C}(\mathrm{M} \mathrm{AX})}=500 \mathrm{~mA}\right.$ ]/ high-voltage $\left[\mathrm{V}_{\mathrm{CE}}(\mathrm{MAX})=50 \mathrm{~V}\right]$ general-purpose Darlington arrays comprising seven silicon NPN Darlington pairs. All units feature open collector outputs and integral diodes for inductive load-transient suppression (e.g., relays). ULN-2003A forms a 16-pin dual in-line package (DIP) and has a 2.7-kohm series base resistor to each Darlington pair. This allows operation to 


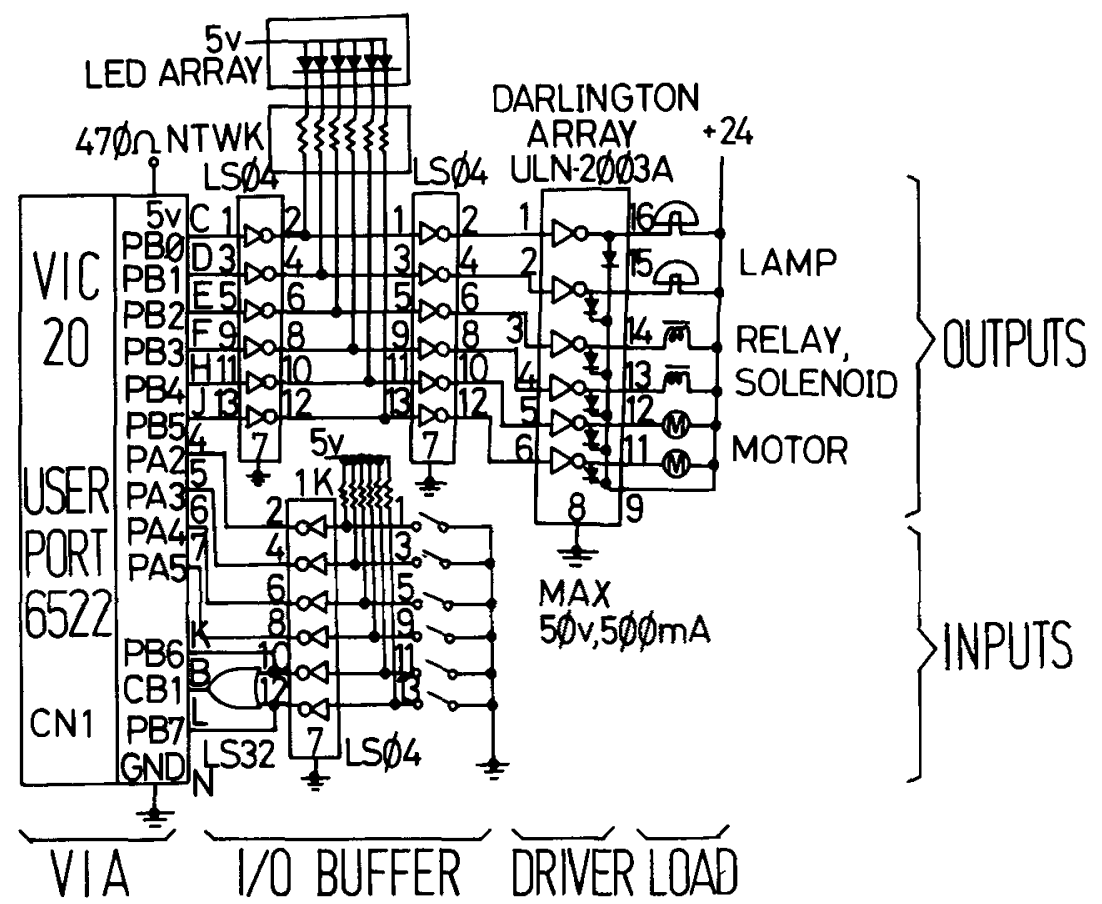

Figure 2. Level 1 interface circuits: Level 1 consists of only four small ICs. The ground of Darlington IC must be connected to the system ground.

be directly compatible with TTL signal. These convenient Darlington arrays enable the system to drive various loads by the TTL logic signals. An experimenter with limited knowledge in electronics can handle the array system as a general "black-box" device. This can be called a sort of "basic black" computer interface (Bailey, 1980; Uttal, 1968). As an example, the loads (incandescent lamps, relays, and dc motors) are pulled up to $\mathrm{V}^{+}(\mathrm{dc} 24 \mathrm{~V})$. Note that the Level 1 interface consists of only four small IC chips.

The following programs indicate the BASIC $1 / 0$ handling procedures $(D D R=$ data direction register; $\mathrm{OR}=$ output register). In order to turn on/off the load,

$\begin{array}{lll}\text { POKE 37138, 15 } & \begin{array}{l}\text { [set "1" to lower 4-bit } \\ \text { (PB0-3) as an output] }\end{array} & \\ \text { then, } & & \\ \text { POKE 37136, 1 } & \text { PB0 turns on } & \text { (OR) } \\ \text { POKE 37136, 2 } & \text { PB1 turns on } & \text { (OR) } \\ \text { POKE 37136,4 } & \text { PB2 turns on } & \text { (OR) } \\ \text { POKE 37136, 8 } & \text { PB3 turns on } & \text { (OR) } \\ \text { POKE 37136,0 } & \text { Any port turns off } & \text { (OR) }\end{array}$

In order to read the data from switch input,

$\begin{array}{lll}\text { PEEK(37136) } & \text { PB6-7 switch detects } & \text { (OR) } \\ \text { PEEK(37137) } & \text { PA2-5 switch detects } & \text { (OR) }\end{array}$

The "memory-mapped" I/O register addresses are listed in Table 1. It should be noted that the I/O handling (soft switch) is quite simple and easy to learn. For a thorough understanding of soft-switch techniques and programmed $\mathrm{I} / \mathrm{O}$ functions, we have to know about the unique function of the interface LSI. The principal device used to transmit signals and data between VIC's CPU (MOS6502A) system and external logic is the MOS6522VIA. Input refers to data transfer from external logic device (e.g., transfer switch) to the 6522VIA, and output refers to data transfer from the 6522VIA to external logic device (e.g., lamp, relay, and so on). Sixteen individually addressable locations are present within a 6522VIA: There are two I/O ports (A and B), two data direction registers (DDR-A and DDR-B), and two control registers associated with each $\mathrm{I} / \mathrm{O}$ port (see Table 1 ). The two $I / O$ ports are accessed as two individually addressable memory locations. These are directly BASIC accessible using PEEKing and POKEing at each $\mathrm{I} / \mathrm{O}$ register location (Osaka, 1979). The data direction register identifies each line of its associated $\mathrm{I} / \mathrm{O}$ port as being dedicated to either input or output mode. A control word ( 0 to 255 ) must be written into each DDR: A " 0 " in a bit position configures the corresponding $\mathrm{I} / \mathrm{O}$ port as an input, whereas a "1" results in an output. For example, if all $\mathrm{I} / \mathrm{O}$ ports are used as output, the memory location of 37138 must be written (that is, DDR-B) " 255 " $\left(2^{0}+2^{1}+\right.$ $\left.2^{2}+2^{3}+2^{4}+2^{5}+2^{6}+2^{7}=255\right)$. This can be done by "POKE 37138, 255." These built-in I/O lines of the VIC, however, appear insufficient for the more complex parallel I/O processing (Osaka, 1980).

\section{Level 2 Communication Interface}

The insufficient $1 / 0$ capability in the Level 1 interface has led to the expansion of the described programmable $\mathrm{I} / \mathrm{O}$ port. Level 2 interface is also easy to build, but it requires some bidirectional bus buffers and an 
Table 1

Memory-Mapped I/O Control Addresses Within a 6255 Versatile Interface Adapter (VIA) Chip

\begin{tabular}{|c|c|c|c|c|c|}
\hline \multirow{3}{*}{$\begin{array}{c}\text { Built-In VIA } \\
\text { User Port } \\
\text { Addresses }\end{array}$} & \multicolumn{2}{|l|}{ VIC-20* } & \multirow{3}{*}{$\begin{array}{c}\text { PET/CBM* } \\
\text { Built-In VIA } \\
\text { User Port } \\
\text { Addresses }\end{array}$} & \multirow[b]{3}{*}{ Register } & \multirow[b]{3}{*}{ Register Function } \\
\hline & \multicolumn{2}{|c|}{ Added VIA** } & & & \\
\hline & $\begin{array}{c}\mathrm{I} / \mathrm{O}-2 \\
\text { Addresses }\end{array}$ & $\begin{array}{c}\text { I/O-3 } \\
\text { Addresses }\end{array}$ & & & \\
\hline 37136 & 38928 & 39952 & 59456 & OR(B) & $\mathrm{I} / \mathrm{O}$ port(B) output register \\
\hline $37137 \dagger$ & 38929 & 39953 & 59457 & OR(A) & $\mathrm{I} / \mathrm{O}$ port $(\mathrm{A})$ output register with handshake \\
\hline 37138 & 38930 & 39954 & $(59458)$ & DDR(B) & I/O port(B) data direction register \\
\hline $37139 \div$ & 38931 & 39955 & 59459 & $\operatorname{DDR}(\mathrm{A})$ & I/O port(A) data direction register \\
\hline 37140 & 38932 & 39956 & 59460 & & H \\
\hline 1 & 1 & 1 & I & $\mathrm{T} 1, \mathrm{~T} 2$ & $\mathrm{~T} 1, \mathrm{~T} 2$ timer \\
\hline 37145 & 38937 & 39961 & 59465 & & \\
\hline 37146 & 38938 & 39962 & 59466 & SR & Shift register \\
\hline 37147 & 38939 & 39963 & 59467 & $\mathrm{ACR}$ & Auxiliary control register \\
\hline 37148 & 38940 & 39964 & 59468 & PCR & Peripheral con trol register \\
\hline 37149 & 38941 & 39965 & 59469 & IFR & Interrupt flag register \\
\hline 37150 & 38942 & 39966 & 59470 & IER & Interrupt enable register \\
\hline $37151 \dagger$ & 38943 & 39967 & 59471 & ORA(A) & I/O port $(A)$ output register \\
\hline
\end{tabular}

Note-Address cross-reference between VIC-20 and PET/CBM microcomputer is shown in decimal notation. *Edge connector is practically compatible among VIC/PET/CBM. **Decoded address is available at VIC's memory expansion connector (CN6): I/O-2 (pin " $T$ ") and $I / O-3$ (pin " $U$ "). †Available without lightpen and joystick.

external VIA chip in addition to the Level 1 interface. Most microcomputers have nonbuffered bus lines at the board edge for further memory expansion (see Figure 1). In Level 2 interface, a memory-mapped $\mathrm{I} / \mathrm{O}$ chip such as VIA $^{1}$ is added for $I / O$ expansion. Figure 3 shows the address bus, data bus, and control bus buffers connecting CPU bus lines and 6522VIA.

VIC.20 has a memory expansion bus connector (CN6) at which CPU bus lines terminate. Since the CPU bus is not buffered, bidirectional data buffers (LS245), address buffers (LS367), and control buffers (LS367) must be interfaced between the CPU bus and VIA. ${ }^{2}$ These buffers must have tristate output. Note that 6522 VIA has a start address of 38928 when the $\mathrm{I} / \mathrm{O} 2$ pin is selected: The I/O2 (and I/O3) line at the edge connector has a decoded address value in the VIC's memory map. Address allocation and function of each register are shown in Table 1.

The "added" VIA controls a total of $16 \mathrm{I} / \mathrm{O}$ lines. As an example, seven loads (PA0-PA6) and six transfer switch inputs (PBO-PB5) are used here for output and input, respectively. Further, the TTL-driven frequencyprogrammable sound generator is connected to the CB2 port. The loads (lamps, relays, solenoids, and dc motors) are driven by Sprague's ULN-2064B high-current $\left[\mathrm{I}_{\mathrm{C}(\mathrm{M} \mathrm{AX})}=1.5 \mathrm{~A}\right] /$ high-voltage $\left[\mathrm{V}_{\mathrm{CE}(\mathrm{MAX})}=50 \mathrm{~V}\right]$ general-purpose Darlington switches. Similar to the ULN-2003A, this 16-pin DIP IC includes suppression diodes and has four independent circuits. PA6 port controls pulse-motor (PF4-36, Nippon Pulse Motor Corporation). The logic driver for the pulse motor (PS-1LD-2) is available with PF4-36 pulse motor (Osaka, 1979). The following list shows the BASIC $\mathrm{I} / \mathrm{O}$ handling pro- gram. Only address values are changed in the memorymap.

In order to turn the load on/off,

POKE 38931, 255 [set "1" to 8-bit (PA0-7) as an output; DDR-A]

then,

$\begin{array}{lll}\text { POKE 38929, 1 } & \text { PA0 turns on } & \text { (OR) } \\ \text { POKE 38929, 2 } & \text { PA1 turns on } & \text { (OR) } \\ \text { POKE 38929, 4 } & \text { PA2 tums on } & \text { (OR) } \\ \text { POKE 38929, 8 } & \text { PA3 turns on } & \text { (OR) } \\ \text { POKE 38929, 16 } & \text { PA4 turns on } & \text { (OR) } \\ \text { POKE 38929, 32 } & \text { PA5 turns on } & \text { (OR) } \\ \text { POKE 38929,64 } & \text { PA6 pulse out } & \text { (OR) } \\ \text { POKE 38929, 0 } & \text { Any port turns off } & \text { (OR) }\end{array}$

In order to read the data,

POKE(38928) PB0-5 switch detects (OR)

\section{COMMUNICATION PROGRAMMING}

Figure 4 shows several sample BASIC programs for Level 1 interface $I / O$ processing. Program 1 generates programmable pulse by using the $\mathrm{PBO}$ port as an output. The duration of the pulse can be varied in steps of 17 msec (i.e., VIC's TI timer units). If the experimenter inputs the value of " 60 " at Statement Number 20 $(\mathrm{K}=60)$, the resulting pulse duration is $1 \mathrm{sec}$. Program 2 generates a sequential pulse for $100 \mathrm{msec}$ to each output port (PBO-5) and then waits for the switch 


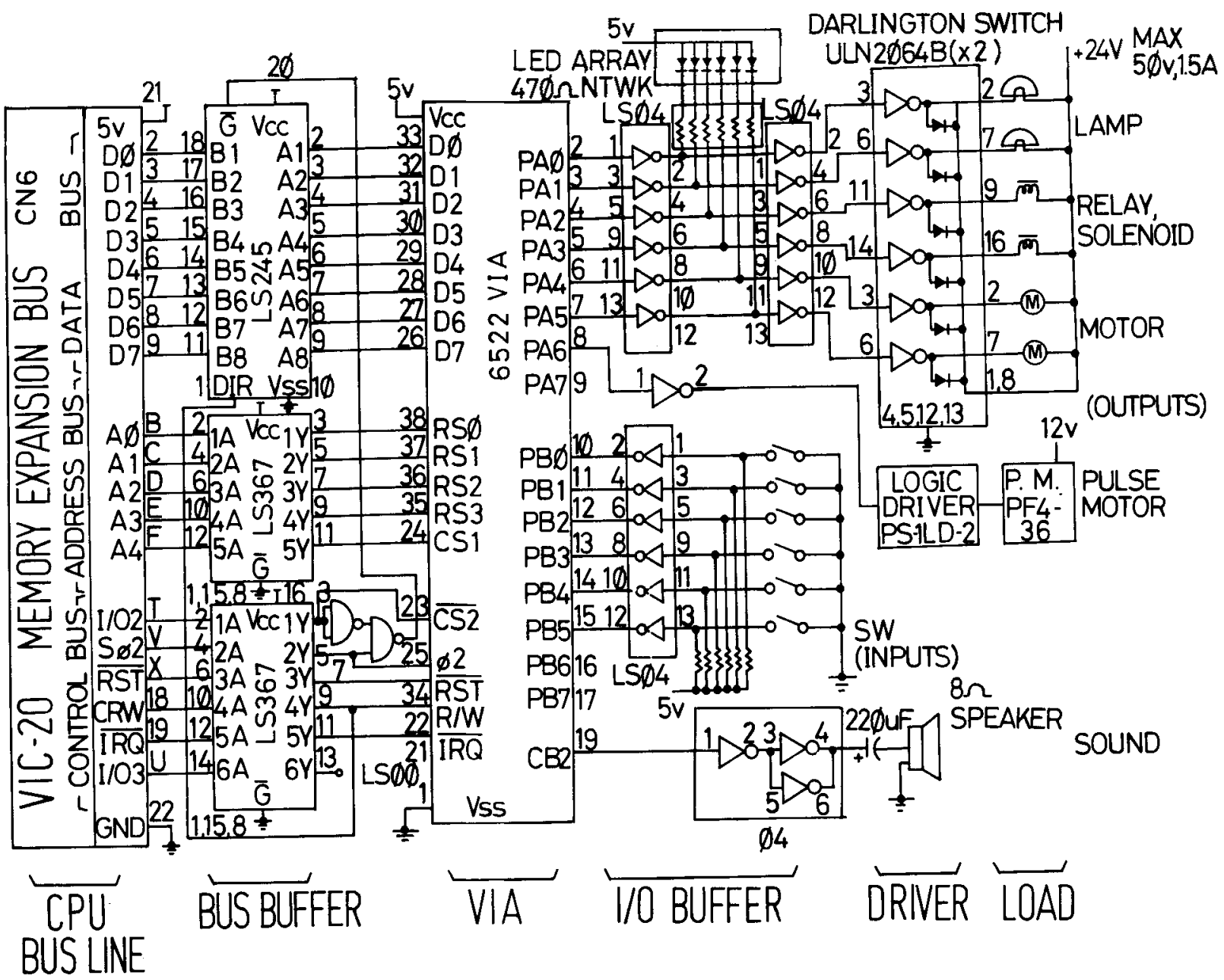

Figure 3. Level 2 interface: The bus buffer and an external "memory-mapped" VIA are added. The ground of Darlington switch IC must be connected to the system ground.

PROGRAM 1

5 A $=37138:$ REM DDR-B LOCATION

6 B $=37136:$ REM I/O PORT-B LOCATION

18 POKE $A, I$ :REM PBQ=OUTPUT

20 INPUT K

30 POKE $B, 1$ :GOSUBiøด:POKE $B, \emptyset$ : GOT020

$109 x=r I$

110 IF TI-X>K THEN RETURN

120 GOT011

PROGRAM 3

\author{
$5 \quad A=37138: B=37136$ \\ 10 POKE A, 1:REM PBQ=OUTPUT \\ 20 $R=I N T(R N D(1) \star 5+1)$ : PRINT $R$ \\ 30 POKE 3,1 :FOR I=I TO 700 : \\ NEXT: POKE $E, \emptyset$ \\ $40 \mathrm{Z}=\mathrm{Tl}$ \\ 50 IF TI-Z>R*60 THEN GOTO 20 \\ 60 GOTO 50
}

$5 A=37138: B=37136$

10 POKE $A, 63:$ REM PB $9-P B 5=0 U T P U T$

20 FOR I $=\varnothing$ TO 5:PRINT I:POKE $B, 2 \uparrow I$ :

FOR $J=1$ TO $100:$ NEXT:POKE $B, \emptyset$ :

NEXT I

$30 \gamma=\operatorname{PEEK}(B)$ : PRINT $Y$ :IF $Y=128$ THEN $5 \emptyset$

40 GOTO $3 \emptyset$

50 PRINT"PB7 SWITCH ACKNOWLEDGEO" : GOTO $2 \emptyset$
$5 \quad A=37138: B=37136$

10 POKE A, 255 : REM PB $\emptyset-P B 7=0 U T P U T$

20 R=INT(RND(1)*7):PRINT R

30 POKE B $, 2 \uparrow R: F O R \quad I=1$ TO 100:NEXT

$4 \emptyset$ POKE $B, \emptyset$

50 GOTO 20

Figure 4. Sample BASIC programs for $1 / O$ processing (Level 1 interface). 
4 REM LEVEL-2 INTERFACE OPERATION

5 P=38928:REM I/O PORT-B LOCATION

$6 X=38931$ :REM OOR-A LOCATION

7 Y $=38929$ : REM I/O PORT-A LOCATION

10 POKE $X, 255:$ REM PAD-PA $=0 U T P U T$

$2 \emptyset$ FOR I= $\emptyset$ TO 7 :PRINT I:POKE $Y, 2 \uparrow I: F O R$ J $=1$ TO $10 \emptyset$ NEXT:POKE Y, $\emptyset$ : NEXT I

25 REM DETECT SWITCH RESPONSE FROM PB PORTS

$30 Z=$ PEEK( $P$ ):PRINT $Z$ :IF $Z<=63$ THEN GOTO $2 \emptyset$

$4 \emptyset$ GOTO $3 \emptyset$

50 STOP

Figure 5. An example BASIC program for $1 / 0$ processing (Level 2 interface).

response. Pushing (grounding) the switch causes a response input (logic "1") to the PB6 or PB7 port. This response is scanned and latched by the VIC's program (PEEK statement). On response, the program restarts. Program 3 generates a 100-msec pulse (PBO port) with a random interpulse interval varying from 1 to $5 \mathrm{sec}$. Further, one of the six output ports (PB0-5) is randomly selected for about $100 \mathrm{msec}$ (Program 4). Figure 5 shows a sample BASIC program for Level 2 interface I/O processing. This program generates a sequential pulse to each output port (PA0-7) and then waits for switch response. On response, the program restarts. This sort of programming appears useful for random and sequential experimental control.

A large portion of the ULN-2003A and 2064B series peripheral power ICs are for relay, solenoid, incandescent lamp, or dc motor interfacing. Using suppression diodes minimizes component count and allows use with inductive loads such as relays or motors. The usual concern for power dissipation/input rush current should be exercised for inductive and lamp loads (Sprague Electric Corporation, 1978).

The interface circuits described here consist of DIP-type ICs: Logic buffers, VIA, power drivers, resistor network, and LED array are all IC packaged. Thus these uniquely designed simple $\mathrm{I} / \mathrm{O}$ interfaces are easy to build: A student unfamiliar with electronics instrumentation may easily be abie to connect the lines among the IC pins designated in the circuits. The cost for the Level 1 and Level 2 interfaces (except loads) is approximately $\$ 20$ and $\$ 36$, respectively.

\section{RFFERENCES}

BALLEY, D. E. "Basic black" renascent: A new wardrobe. Behavior Research Methods \& Instrumentation, 1980, 12, 96-102. Commodone Business Machines Japan, Inc. VIC-1001 user's manual. Tokyo: Author, 1981.

Osaka, N. A microprocessor-based real-time BASIC laboratory: A pulse-motor controlled visual stimulator. Behavior Research Methods \& Instrumentation, 1979, 11, 549-552.

Osaka, N. An inexpensive microcomputer peripheral I/O expansion. Behavior Research Methods \& Instrumentation, 1980, 12, 344-345.

OsakA, N. [A microcomputer-based BASIC laboratory instrumentation for psychologyl (in Japanese). Kyoto: Nakanishiya, 1982.

Parks, E. R. A general-purpose microcomputer configuration for controlling experiments. Behavior Research Methods \& Instrumentation, 1978, 10, 480-484.

SidowgkI, J. B. Microcomputers in psychology. Behavior Research Methods \& Instrumentation, 1978, 10, 463-467.

Sprague Electric Conporation. Sprague integrated circuits data book 1 (SWTH-500). North Adams, Mass: Author, 1978.

UTTAL, W. R. "Basic black" in computer interfaces for psychological research. Behavior Research Methods \& Instrumentation, 1968, 1, 35-40.

\section{NOTES}

1. Intel's 8255C programmable peripheral interface (PPI), Motorola's MC6821 peripheral interface adapter (PIA), and Zilog's parallel input/output (PIO) are also applicable.

2. More detailed IC pin configuration information can be obtained from the data book: Commodore Business Machines, INc., MCS6522 Versatile Interface Adapter (preliminary data sheet, November 1977), and Texas Instruments, Inc., The Bipolar Digital Integrated Circuits Data Book: TTL Bipolar Memory and Interface Circuits (Dallas, Texas, 1978).

(Received for publication June 29, 1981; revision accepted October 13,1981 .) 